\title{
BMJ Open The impact of HPV vaccination on future cervical screening: a simulation study of two birth cohorts in Denmark
}

\author{
Mie Sara Hestbech, ${ }^{1}$ Elsebeth Lynge, ${ }^{2}$ Jakob Kragstrup, ${ }^{1}$ Volkert Siersma, ${ }^{1}$ \\ Miguel Vazquez-Prada Baillet, ${ }^{2}$ John Brodersen ${ }^{1}$
}

To cite: Hestbech MS,

Lynge E, Kragstrup J, et al. The impact of HPV

vaccination on future cervical screening: a simulation study of two birth cohorts in Denmark. BMJ Open 2015;5: e007921. doi:10.1136/ bmjopen-2015-007921

- Prepublication history and additional material is available. To view please visit the journal (http://dx.doi.org/ 10.1136/bmjopen-2015007921).

Received 11 February 2015 Revised 29 June 2015 Accepted 30 June 2015

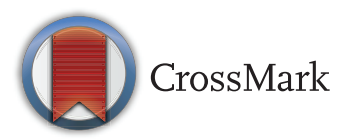

${ }^{1}$ The Research Unit for General Practice and Section of General Practice, Department of Public Health, University of Copenhagen, Copenhagen, Denmark

${ }^{2}$ Department of Public Health, Center of Epidemiology and Screening, University of Copenhagen, Copenhagen, Denmark

Correspondence to Dr Mie Sara Hestbech; mie.hestbech@sund.ku.dk

\section{ABSTRACT}

Objectives: To explore the interplay between primary and secondary prevention of cervical cancer by estimating future screening outcomes in women offered human papillomavirus (HPV) vaccination when they were sexually naïve.

Design: Estimation of outcome of liquid-based cytology screening for a post-HPV vaccination cohort using pre-vaccination screening data combined with HPV vaccination efficacy data reported in the literature. Setting: Denmark.

Data: The number of screening diagnoses at first screen in a pre-vaccination birth cohort was multiplied by reported risk reductions expected for women who were vaccinated for HPV before sexual debut. All identified studies were reviewed by two authors, and weighted pooled estimates of vaccine efficacies were used.

Main outcome measures: Proportions of positive and false-positive cervical cytologies and positive predictive value (PPV) were calculated using cervical intraepithelial neoplasia (CIN) grade $2+$ and $3+$ as cutoff values.

Results: The proportion of positive screening tests was reduced from $8.7 \%$ before vaccination to $6.5 \%$ after vaccination, and the proportion of false-positive screening tests using CIN2+ as a cut-off was reduced from $5.5 \%$ pre-vaccination to $4.3 \%$ post-vaccination, and using $\mathrm{CIN} 3+$ as a cut-off from $6.2 \%$ to $4.7 \%$. PPVs were reduced from $23 \%$ to $19 \%$ (cut-off CIN2+), and from $14 \%$ to $12 \%$ (cut-off CIN3+).

Conclusions: In our calculations, the proportion of positive screening results with liquid-based cytology will be reduced as a consequence of HPV vaccination, but the reduction is small, and the expected decline in PPV is very limited. In this situation, the information general practitioners will have to provide to their patients will be largely unchanged.

\section{INTRODUCTION}

Screening for cervical cancer has been gradually implemented in high-income countries since the $1960 \mathrm{~s}^{12}$ and has been followed by a significant reduction in cervical cancer

\section{Strengths and limitations of this study}

- In a few years, several countries will be facing a so far unknown situation within the area of prevention, where a growing population of women is offered two types of prevention against the same type of cancer: human papillomavirus (HPV) vaccination and cervical cancer screening. This research paper explores this interplay between primary and secondary prevention of cervical cancer.

- We focus on the following outcomes: Proportions of positive and false-positive cervical cytologies and positive predictive value (PPV) of cervical screening. These outcomes are important for answering the question: How should general practitioners change the information they provide to women regarding the expected outcomes of the screening?

- The calculations in this paper are based on a combination of historical data from registers and HPV vaccination efficacy data reported in the literature. It is a limitation to the study that we have to rely on these types of data. However, as it is, there are still no clinical data available that report screening outcomes from screening of women who were HPV vaccinated before sexual debut. Therefore, we have to rely on analyses such as those presented in this research paper.

incidence and mortality. This reduction is assumed to be largely attributable to screening, ${ }^{3-5}$ but screening also leads to unintended harms such as false-positive results, overdiagnosis and overtreatment. It has been calculated that for each prevented death from cancer, around 1000 women will undergo a biopsy. ${ }^{5}$ Another study has shown that for each prevented case of cervical cancer, 6-8 women will undergo treatment for pre-cancerous lesions. ${ }^{6}$ Human papillomaviruses (HPVs) are a necessary cause of cervical cancers. ${ }^{7}$ In 2006, the quadrivalent HPV vaccine Gardasil (Merck, Whitehouse Station, New Jersey, USA) was licensed for 
use. $^{8}$ This vaccine prevents infection from HPV types 6 and 11 , which cause the majority of anogenital warts, plus types 16 and 18, which are responsible for approximately $70 \%$ of cervical cancers. ${ }^{9}$ Randomised controlled trials (RCTs) with Gardasil have shown up to $98 \%$ efficacy on vaccine-type-specific, high-grade cervical intraepithelial neoplasia (CIN) in young women not yet infected with HPV at the time of first vaccination. ${ }^{10} 11$

These results indicate that HPV vaccination will reduce the prevalence of cervical cancer significantly in generations that are vaccinated as HPV naïve, which is basically before the start of sexual life. The presumed vaccine effect is, however, not complete and continued screening in some form is still widely recommended. ${ }^{712}{ }^{13}$ With the future combination of primary and secondary prevention of cervical cancer screening, the balance between benefits and harms of screening may change. One of the harms of screening is false-positive results. Owing to the reduced incidence of cervical cancer, the predictive value of a positive screening may be reduced and the proportion of false-positive results could increase.

The aim of this study was to test these hypotheses and to explore the interplay between primary and secondary prevention of cervical cancer in future primary healthcare by focusing on one of the important aspects of a screening programme: the frequency of positive screening outcomes. To what extent should general practitioners (GPs) change the information they provide to women regarding the expected outcomes of the screening? To predict the future outcomes of screening, we combined pre-vaccination screening data from Denmark and previously reported HPV vaccination efficacy data.

\section{METHODS}

\section{Setting}

We used Denmark as a case because of the unique access to historical data from registers. In Denmark, the National Board of Health recommends screening with liquid-based cytology (LBC) every 3 years among women aged 23-49 years, and every 5 years in the age group 50-64. ${ }^{14}$ Until 2007, the recommendation was screening every 3 years from age 23 to 59 with conventional cytology. Screening samples are taken by GPs.

Gardasil was licensed for use in Denmark in October 2006. From 1 October 2008, women born between 1993 and 1995 were offered free Gardasil vaccination in a catch-up programme, and from 1 January 2009, free vaccination was offered to all girls turning 12 years as a part of the national childhood immunisation programme. Approximately $80 \%$ of these birth cohorts have received all three doses of the vaccine. ${ }^{15}$

\section{Populations}

We followed two cohorts of women. First, women born in 1982, turning 23 years and invited to screening for the first time in 2005. Few of these women are expected to have been HPV vaccinated as the vaccine was marketed in October 2006. Second, we simulated data for women born in 1993. They form the first cohort that can be considered, with some modification, to have been sexually naive at the time of vaccination, and they will turn 23 and enter the screening programme in 2016. It is reasonable to assume that Danish women born in 1982 and born in 1993 are similar when it comes to their history risk of HPV infection and cervical dysplasia, had it not been for the HPV vaccine. Sociodemographic data support this assumption, since the proportion of 20-24 year-old women in Denmark with completed high-school education has thus been fairly stable for the past 10 years. ${ }^{16}$ Moreover, studies of sexual habits among young Danish women suggest that the age of sexual debut has been fairly stable over the past 30 years with a mean age of sexual debut around $16 .{ }^{17-19}$

\section{Screening data}

Data on screening diagnoses were obtained from an existing database described previously. ${ }^{20}$ The Danish National Pathology Data Bank (Patobank; established in 1978) ${ }^{21}$ and the National Health Service Register (NHSR; established in 1990) ${ }^{22}$ were combined to retrieve complete information on cervical screening. In the Patobank, a sample is coded with SNOMED codes ${ }^{21}$ for topography and morphology. NHSR is a payment register including information on the service provided but without diagnostic codes. The outcome of a sample was defined by the most severe SNOMED morphology code. The term 'missing' was used for samples known only from the NHSR. We excluded the Copenhagen County since it was the only area in Denmark with incomplete Patobank data in 2005.

Cervical cytology was classified according to the Bethesda system based on SNOMED codes as normal cells; ASCUS (Atypical Squamous Cells of Undetermined Significance); LSIL (Low-grade Squamous Intraepithelial Lesion); HSIL (High-grade Squamous Intraepithelial Lesion); ASCH (Atypical Squamous Cells, Cannot Rule Out HSIL) and AGC (Atypical Glandular Cells); unsatisfactory and missing. A sample was considered positive if the diagnosis was ASCUS or a more severe abnormality (ASCUS+). In the case of ASCUS/LSIL, the Danish guideline in 2005 was cytological follow-up after 6 months. If the following cytology revealed abnormality, a biopsy was indicated. In the case of HSIL/ASCH/AGC, the guideline was immediate biopsy. ${ }^{23}$

Cervical histology (biopsies) was classified as normal tissue, CIN1, CIN2, CIN3 and above (cases coded as carcinoma in situ and carcinomas), and missing.

\section{HPV vaccination efficacy data}

We searched the literature for studies reporting estimates for efficacy of the quadrivalent HPV vaccine on cytological and histological cervical abnormalities in HPV-naïve women. We were interested in effects on all-type HPV abnormalities (not vaccine-type-specific 
effects), as these translate directly to clinically relevant reductions in dysplasia. PubMed was searched using 'HPV vaccine' as a MESH term in combination with 'studies, case control', 'studies, cohort' and 'randomized controlled trial'. Reference lists in papers were manually checked for other relevant studies. Experts in the field were contacted to check for possible additional references. We excluded one ecological study ${ }^{24}$ because the same population was covered by a data linkage study. ${ }^{25}$ All identified studies were reviewed by two authors.

We extracted data on vaccine efficacy for women vaccinated as HPV naive, or proxies for this group. Consequently, we used results for the youngest age strata when results were stratified according to age at vaccination in the population-based studies. To assure comparability, we always used unadjusted/crude estimates in our further calculations.

\section{Analysis}

The birth cohort of 1982 included a total of 26082 women in 2005, excluding the Copenhagen County. Of these women, $7750 \quad(=30 \%)$ had their first cytology sample prior to the age of 23 years; $10205(=39 \%)$ at the age of 23 years; and $4702(=18 \%)$ after the age of 23 years. By 2010, 3425 (=13\%) still had no cytology sample registered. Among the 7750 women with a cytology sample prior to the age of 23 years, 1250 $(=16 \%)$ of the samples had no diagnosis, meaning that they were known only from the NHSR. For the 14907 women with a first cytology sample at or after the age of 23 years, only $148(=1 \%)$ of the samples had no diagnosis. We therefore excluded women with a cytology sample prior to the age of 23 years. In total, 10205 women had their first cytology sample at the age of 23 years; 1870 at 24 years; 928 at 25 years and 1904 at 26 years or above. The proportions of abnormal outcomes varied little across the age groups with the percentages of ASCUS+ being approximately 8\%, 9\%, 9\% and $9 \%$, respectively, and the percentages of CIN2+ being approximately $1 \%, 2 \%, 2 \%$ and $2 \%$, respectively. Consequently, we analysed women with a first cytology sample at the age of 23 years and above as one group.

For women born in 1982 and having their first cytology sample at the age of 23 years or above, we recorded the outcome of this sample and the follow-up according to Danish guidelines. For women with ASCUS/LSIL, we followed the outcome of the next cytology, and if that was ASCUS+, we followed the outcome of the histology. For women with HSIL/ ASCH/AGC, we followed the outcome of the histology. Follow-up ceased 3 years after the first cytology sample was taken. Conventional cytology was the most widely used screening test in Denmark in 2005, which was reflected in an unsatisfactory rate of $4.7 \%$ in our data. However, from 2014 onwards, all cytology in Denmark will be liquid based and the unsatisfactory rate is expected to be very low. ${ }^{26}$ One per cent of the first cytology samples in our 2005 data were known only from the NHSR, but for later years all samples are included in the Patobank with a diagnosis. For these reasons, we used only the number of samples with a known diagnosis as the basis for the prediction of screening outcome in HPV-vaccinated women.

When more than one estimate of vaccine efficacy for a specific outcome was identified in the literature, a meta-analysis was done, using the random effects model with a restricted maximum likelihood (REML) estimation method, to obtain a weighted pooled estimate of the vaccine efficacies. Pooling of the different measures of efficacy is justified because of the relatively low outcome incidence in the background populations. The expected outcome of screening for a cohort of women where $80 \%$ were HPV vaccinated as HPV naïve was estimated by multiplying the observed screening outcome for women born in 1982 with $80 \%$ of the pooled estimate of vaccine efficacy retrieved from the literature. In time, herd immunity is expected to compensate for the incomplete coverage, but not for the first decades. Sensitivity analyses were conducted using 'best case' and 'worst case' estimates of vaccine efficacy.

On the basis of the follow-up data, women with an ASCUS+ test at screening could be divided into three groups: true positives, false positives and unresolved cases without a complete follow-up (unsatisfactory and missing samples). We used two cut-offs for definition of true positives, CIN2+ and CIN3+, respectively. The positive predictive value (PPV) of screening was calculated in two ways. First, true positives as a proportion of all ASCUS+ and second, true positives as a proportion of resolved ASCUS+ (=true positives+false positives). We assumed the proportion of unresolved ASCUS+ samples to remain the same over time.

\section{RESULTS}

All vaccine efficacy estimates were reported with relatively broad CIs (table 1) (see online supplementary table, appendix). In our meta-analysis, Gardasil vaccination of HPV-naïve women was associated with a $32 \%$ reduction in proportion of women with ASCUS+, a 22\% reduction in cervical biopsies (based on one study only), and reductions of $49 \%$ and $47 \%$ in CIN2+ and CIN3+, respectively. Using these efficacy estimates on the Danish population, we expect the proportion of women with ASCUS+ to decrease from the previous $8.7 \%$ to a future $6.4 \%$ among young women entering the screening programme (table 2). The proportion of women with CIN2+ at first screen was expected to decrease from $1.6 \%$ to $1.0 \%$, and the proportion with CIN3+ from $1.0 \%$ to $0.6 \%$. Including only resolved samples, the proportion of true positives with CIN2+ as a cut-off was expected to decrease from $1.7 \%$ to $1.0 \%$, and the proportion of false positives from $5.5 \%$ to $4.3 \%$ (table 3 ). These changes were reflected in a change in the PPV from $23 \%$ to $19 \%$. In a similar calculation, the PPV using CIN3+ as a cut-off was expected to change from $14 \%$ to $12 \%$. 


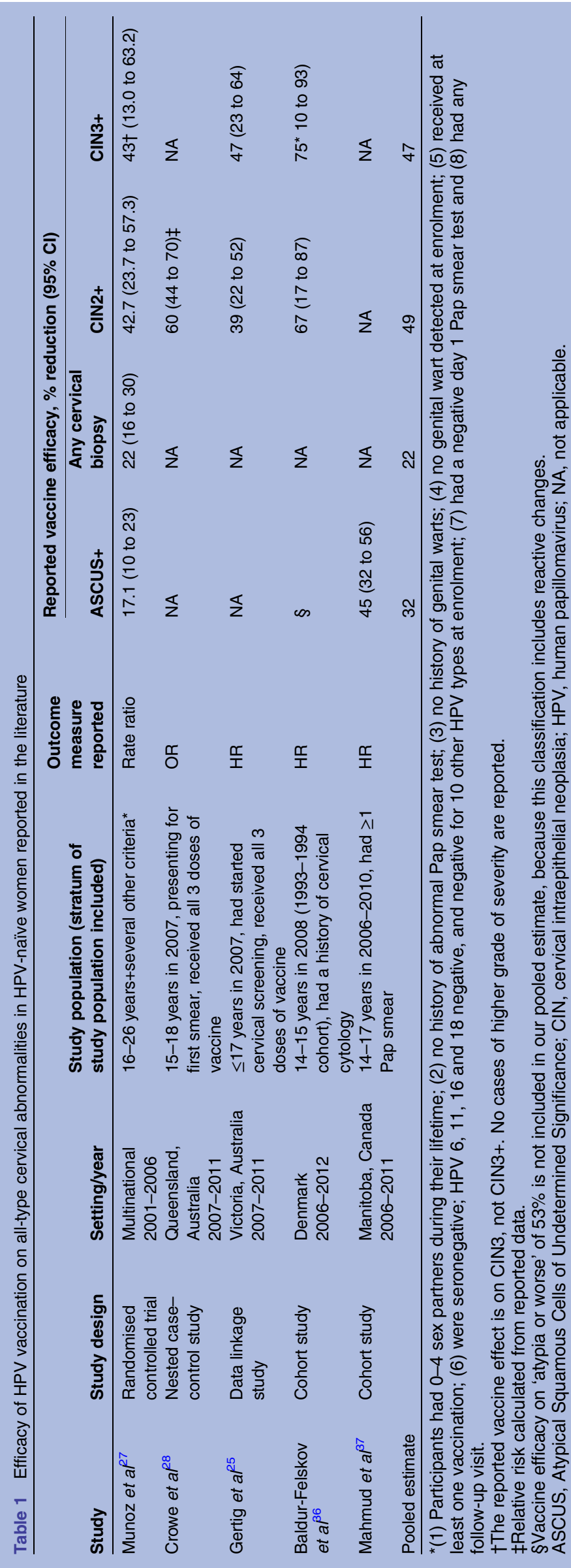

Sensitivity analyses using extreme values of vaccine efficacies resulted in a 'best case vaccine effect' estimate of PPV of $16.7 \%$ using CIN2+ as a cut-off and 9\% using CIN3+ as a cut-off and a 'worst case vaccine effect' estimate of $17.4 \%$ using CIN2+ as a cut-off and $10.4 \%$ using CIN3+ as a cut-off.

\section{DISCUSSION}

Our study showed a surprisingly small effect of HPV vaccination on the expected outcome in young women participating in cervical screening for the first time. We found minor declines both in the number of truepositive screening tests and in the number of falsepositive screening tests. Using CIN2+ as a cut-off, the pre-vaccination PPV of $23 \%$ was expected to decrease to a post-vaccination PPV of $19 \%$. This means that prevaccination, $23 \%$ women with an abnormal screening result had an underlying histological abnormality of CIN2 or more severe and $77 \%$ had a false-positive screening result. In our calculations, the corresponding post-vaccination proportions would be $19 \%$ and $81 \%$. Our findings are contrary to our a priori hypotheses, as we expected to find more pronounced effects of HPV vaccination on the outcomes of cervical cancer screening.

There are a number of limitations pertaining to the evidence on vaccine efficacy. First, data were available from only the FUTURE trials, ${ }^{27}$ and from four population-based studies. Even well-conducted population-based studies will have a higher chance of being biased than RCTs. On the other hand, the RCTs included selected populations and the study populations were treated according to protocols different from those found in screening programmes. Therefore, we also decided to include the population-based studies in our meta-analysis. Results are obviously determined by the parameters of the model. However, sensitivity analysis substituting the pooled estimates with extreme values of vaccine efficacy resulted in quite similar estimates. Second, the studies included in our analysis had follow-up times of $<5$ years and used surrogate outcomes. Thus, there was a lack of evidence on the length of vaccine protection and actual protection against cervical cancer; the possible long-term effects of the vaccine remain to be seen. It should, however, be emphasised that this study focuses on the clinical situation shortly after implementation of the HPV vaccination, not on the long-term consequences. Finally, in the populationbased studies, HPV naïvety was only approximated. In the two Australian studies, the study populations were either vaccinated before the age of 17 years $^{25}$ or women presenting for their first smear ${ }^{28}$ (at the age of 18 years or sexual debut, whichever came first). From the Canadian and Danish studies, we included the age groups $15-17$ and $12-16$ years, respectively, to approximate HPV-naive cohorts. This might underestimate vaccine efficacy. Nevertheless, the first vaccinated 
Table 2 Observed pre-HPV vaccination outcome of first cytology sample in a cohort of women who turned 23 years, and expected outcome for a similar post-HPV vaccination cohort

\begin{tabular}{|c|c|c|c|c|c|}
\hline & $\begin{array}{l}1982 \text { Cohort, } \\
\text { number of } \\
\text { first samples } \\
\mathrm{N}=14907\end{array}$ & $\begin{array}{l}\text { Proportion of } \\
\text { first samples } \\
\mathrm{N}=14907(\%)\end{array}$ & $\begin{array}{l}\text { Proportion of first } \\
\text { samples, excluding } \\
\text { unsatisfactory and } \\
\text { missing } \\
\mathrm{N}=14062(\%)\end{array}$ & $\begin{array}{l}80 \% \text { of } \\
\text { vaccine } \\
\text { efficacy }\end{array}$ & $\begin{array}{l}\text { Expected } \\
\text { abnormality } \\
\text { post-vaccination } \\
\text { proportiont (\%) }\end{array}$ \\
\hline \multicolumn{6}{|l|}{ Cytology } \\
\hline Normal & 12844 & 86.2 & 91.3 & & \\
\hline ASCUS+ & 1218 & 8.2 & 8.7 & 25.6 & 6.4 \\
\hline Unsatisfactory & 697 & 4.7 & NR & & \\
\hline Missing & 148 & 1.0 & NR & & \\
\hline \multicolumn{6}{|l|}{ After ASCUS+ } \\
\hline Normal cytology & 452 & 3.0 & 3.2 & & \\
\hline $\begin{array}{l}\text { Unsatisfactory/missing cytology } \\
\text { code }\end{array}$ & 40 & 0.3 & 0.3 & & \\
\hline Histology & 575 & 3.9 & 4.1 & 17.6 & 3.4 \\
\hline Inadequate follow-up & 151 & 1.0 & 1.1 & & \\
\hline \multicolumn{6}{|l|}{ Histology } \\
\hline Normal/CIN1/unsatisfactory $\ddagger$ & 312 & 2.1 & 2.2 & & \\
\hline CIN2+ & 230 & 1.5 & 1.6 & 39.2 & 1.0 \\
\hline CIN3+ & 141 & 0.9 & 1.0 & 37.6 & 0.6 \\
\hline Missing histology code & 33 & 0.2 & 0.2 & & \\
\hline \multicolumn{6}{|c|}{$\begin{array}{l}\text { `Efficacy estimate reduced to account for vaccination coverage in the female population of } 80 \% . \\
\text { tCalculated based on all first samples, excluding unsatisfactory and missing. } \\
\text { f29 Women without CIN and/or cervical cancer, but with other non-mormal codes. } \\
\text { ASCUS, Atypical Squamous Cells of Undetermined Significance; CIN, cervical intraepithelial neoplasia; HPV, human papillomavirus; NR, not } \\
\text { reported. }\end{array}$} \\
\hline
\end{tabular}

cohorts entering screening in Denmark from 2016 onwards were vaccinated in a catch-up programme at the age of $13-15$ years, and were therefore presumably not completely HPV-naive cohorts either. ${ }^{29}$

There are also limitations pertaining to the screening data. First, we had to exclude 7750 women with a cytology sample prior to the age of 23 years because 1250 of these women had no diagnosis recorded in the Patobank. The excluded women might constitute a highrisk group, although this was not indicated in the data from the 6500 women with a diagnosis. Anyhow, if the 7750 excluded women constituted a high-risk group, it would mean that our estimate of the expected proportion of women with ASCUS+ would be a conservative one. Second, the pre-vaccination cohort born in 1982 was predominantly screened with conventional cytology, which has a higher proportion of unsatisfactory samples than LBC. We accounted for this by excluding the unsatisfactory samples from our calculations.

To the best of our knowledge, this is the first study investigating the impact of HPV vaccination on PPV of cervical screening. Our calculated pre-vaccination PPV for CIN2+ was well in accordance with the PPV of $21 \%$ previously estimated for the Danish screening programme. ${ }^{30}$ We calculated PPV for the cut-off values CIN2+ and CIN3+, and in the interpretation of our results it is important to keep in mind that the progression rates to invasive cancer have been estimated to be only $5 \%$ for CIN2 ${ }^{31}$ and $31 \%$ for CIN3. ${ }^{32}$
The relatively small effect of HPV vaccination on screening outcomes in our calculations was explained by the HPV vaccine efficacy data and the distribution of HPV type $16 / 18$ in cervical lesions. The FUTURE trials ${ }^{33}$ found an almost complete protection against vaccine HPV-type associated CIN2+ in HPV-naive women. Combined with the estimate of approximately $40 \%$ of CIN2 and $65 \%$ of CIN3 being caused by HPV 16 and $18,{ }^{34} 35$ we would expect efficacy within this range. The all-HPV-type efficacy on CIN2+ was $43 \%$ in the FUTURE trials, ${ }^{27}$ and with varying outcomes from the populationbased studies, the estimate of $49 \%$ from our meta-analysis was close to that of the FUTURE trials alone.

The difference in vaccine efficacy between cytology and histology reflects that the progression rates of HPV 16 and 18 are higher than those of other high-risk HPV types. Thus, the proportion of HPV 16 and 18 is significantly higher in histological abnormalities than in cytological abnormalities, and it increases with increasing severity of CIN. ${ }^{34}{ }^{35}$ Therefore, the reduction in abnormalities after HPV vaccination is expected to increase with increasing severity of abnormality, and thus the proportion of abnormal cytology outcomes with an underlying high-grade CIN is expected to be lower for vaccinated than for non-vaccinated women. This would contribute to a drop in PPV, and this is why we expected a larger decline in PPV than we found.

From the GP's perspective, our study indicated that the information regarding interpretation of an abnormal 


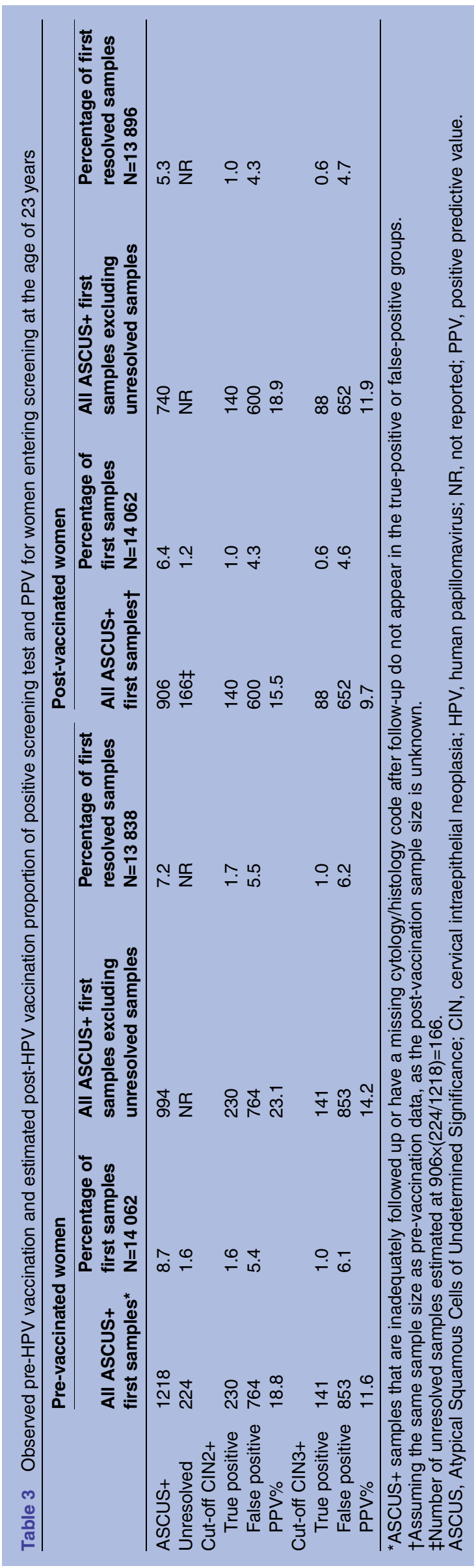

cytology to be provided to young HPV-vaccinated women undergoing cervical screening with LBC will be largely the same as that provided to previous generations of unvaccinated women. In other words, the way a positive cytology should be perceived, as more or less likely to represent an underlying histological abnormality, will not change. This is not a satisfactory situation, neither for the women nor for their GPs, given that HPV vaccination is expected to decrease the risk of cervical cancer by $70 \%$. Changes in the modalities of cervical screening and new screening modes with higher PPVs are therefore warranted for these generations of women.

Acknowledgements The authors would like to thank Bruno Heleno, MD, for valuable input in the process of forming and writing this paper.

Contributors JB conceived the idea for the project. MSH drafted the protocol in cooperation with EL, JK and JB. MV-PB extracted the data. MSH analysed the data and drafted the manuscript. VS contributed to the analysis with statistical expertise. All authors contributed to revisions with important intellectual content. They had full access to all data (including statistical reports and tables) in the study and take responsibility for the integrity of the data and the accuracy of the data analysis. MSH is the guarantor.

Funding MSH is partially funded by Helsefonden, an independent charity.

Competing interests EL is undertaking a comparative study of new-generation HPV assays, involving collaboration with Roche, Genomica, Qiagen and Hologic/Gen-Probe, a self-sampling pilot involving collaboration with Genomica and BD, and she has served as an unpaid advisor to Hologic/ Gen-Probe and Norchip.

Provenance and peer review Not commissioned; externally peer reviewed.

Data sharing statement The full data set including a flow chart of cytological diagnoses in the 1982 cohort is available from the corresponding author; mie.hestbech@sund.ku.dk.

Open Access This is an Open Access article distributed in accordance with the Creative Commons Attribution Non Commercial (CC BY-NC 4.0) license, which permits others to distribute, remix, adapt, build upon this work noncommercially, and license their derivative works on different terms, provided the original work is properly cited and the use is non-commercial. See: http:// creativecommons.org/licenses/by-nc/4.0/

\section{REFERENCES}

1. Lynge E. [Cervical cancer screening]. Ugeskr Laeger 2002;164:171-3.

2. Peirson L, Fitzpatrick-Lewis D, Ciliska D, et al. Screening for cervical cancer: a systematic review and meta-analysis. Syst Rev 2013;2:35.

3. Sasieni P, Adams J. Effect of screening on cervical cancer mortality in England and Wales: analysis of trends with an age period cohort model. BMJ 1999;318:1244-5.

4. Peto J, Gilham C, Fletcher O, et al. The cervical cancer epidemic that screening has prevented in the UK. Lancet 2004;364:249-56.

5. Raffle AE, Alden B, Quinn M, et al. Outcomes of screening to prevent cancer: analysis of cumulative incidence of cervical abnormality and modelling of cases and deaths prevented. BMJ 2003;326:901.

6. Barken SS, Rebolj M, Andersen ES, et al. Frequency of cervical intraepithelial neoplasia treatment in a well-screened population. Int J Cancer 2012;130:2438-44.

7. Carter JR, Ding Z, Rose BR. HPV infection and cervical disease: a review. Aust N Z J Obstet Gynaecol 2011;51:103-8.

8. Sander BB, Rebolj M, Valentiner-Branth $\mathrm{P}$, et al. Introduction of human papillomavirus vaccination in Nordic countries. Vaccine 2012;30:1425-33

9. Munoz N, Bosch FX, de Sanjosé S, et al. Epidemiologic classification of human papillomavirus types associated with cervical cancer. N Engl J Med 2003;348:518-27.

10. Dillner J, Kjaer SK, Wheeler CM, et al. Four year efficacy of prophylactic human papillomavirus quadrivalent vaccine against low grade cervical, vulvar, and vaginal intraepithelial neoplasia and anogenital warts: randomised controlled trial. BMJ 2010;341:c3493. 
11. Kjaer SK, Sigurdsson K, Iversen OE, et al. A pooled analysis of continued prophylactic efficacy of quadrivalent human papillomavirus (Types 6/11/16/18) vaccine against high-grade cervical and external genital lesions. Cancer Prev Res (Phila) 2009;2:868-78.

12. Danish Centre for Health Technology Assessment. Reduction in the risk of cervical cancer by vaccination against human papillomavirus (HPV)—a health technology assessment. Copenhagen: National Board of Health. Ref Type: Report, 2007.

13. Stanley M. Human papillomavirus vaccines versus cervical cancer screening. Clin Oncol (R Coll Radiol) 2008;20:388-94.

14. [Screening for cervical cancer-recommendations]. Copenhagen: National Board of Health, 2012.

15. Statens Serum Institut. HPV-vaccination - tilslutning 2012. EPI-NYT 2013;20. http://www.ssi.dk/Aktuelt/Nyhedsbreve/EPI-NYT/2013/Uge $\% 2020 \% 20-\% 202013$.aspx

16. http://www.statistikbanken.dk/statbank5a/default.asp?w=1280. 19-1-2015.

17. Olesen TB, Jensen KE, Munk C, et al. ["Liva"-population survey of female sexual habits]. Ugeskr Laeger 2010;172:3254-9.

18. Jorgensen MJ, Maindal HT, Christensen KS, et al. Sexual behaviour among young Danes aged 15-29 years: a cross-sectional study of core indicators. Sex Transm Infect 2015;91:171-7.

19. Graugaard CL, Rasmussen B, Boisen KA. [Sexual knowledge, attitudes and behavior among young Danes. A questionnaire study]. Ugeskr Laeger 2002;164:4810-14.

20. Dugue PA, Lynge E, Bjerregaard B, et al. Non-participation in screening: the case of cervical cancer in Denmark. Prev Med 2012;54:266-9.

21. Bjerregaard B, Larsen OB. The Danish Pathology Register. Scand J Public Health 2011;39(7 Suppl):72-4.

22. Olivarius NF, Hollnagel $H$, Krasnik A, et al. The Danish National Health Service Register. A tool for primary health care research. Dan Med Bull 1997;44:449-53.

23. [Screening for cervical cancer-recommendations]. Copenhagen: National Board of Health, 2007.

24. Brotherton JM, Fridman M, May CL, et al. Early effect of the HPV vaccination programme on cervical abnormalities in Victoria, Australia: an ecological study. Lancet 2011;377:2085-92.

25. Gertig DM, Brotherton JM, Budd AC, et al. Impact of a population-based HPV vaccination program on cervical abnormalities: a data linkage study. BMC Med 2013;11:227.
26. Danish Qualitydatabase for Cervical Cancer Screening. Danish Qualitydatabase for Cervical Cancer Screening (DKLS) Yearly Report 2012. 2013.

27. Munoz N, Kjaer SK, Sigurdsson K, et al. Impact of human papillomavirus (HPV)-6/11/16/18 vaccine on all HPV-associated genital diseases in young women. J Natl Cancer Inst 2010;102:325-39.

28. Crowe E, Pandeya N, Brotherton JM, et al. Effectiveness of quadrivalent human papillomavirus vaccine for the prevention of cervical abnormalities: case-control study nested within a population based screening programme in Australia. BMJ 2014;348:g1458.

29. [Study of 11-15-year-olds lifestyle and health behaviour 19972008]. 2010. Danish National Board of Health.

30. Ronco $\mathrm{G}$, van Ballegooijen $\mathrm{M}$, Becker $\mathrm{N}$, et al. Process performance of cervical screening programmes in Europe. Eur $J$ Cancer 2009;45:2659-70.

31. Ostor AG. Natural history of cervical intraepithelial neoplasia: a critical review. Int J Gynecol Pathol 1993;12:186-92.

32. McCredie MR, Sharples KJ, Paul C, et al. Natural history of cervical neoplasia and risk of invasive cancer in women with cervical intraepithelial neoplasia 3: a retrospective cohort study. Lancet Oncol 2008;9:425-34.

33. FUTURE II Study Group. Quadrivalent vaccine against human papillomavirus to prevent high-grade cervical lesions. N Engl J Med 2007;356:1915-27.

34. Kjaer SK, Breugelmans G, Munk C, et al. Population-based prevalence, type- and age-specific distribution of HPV in women before introduction of an HPV-vaccination program in Denmark. Int $J$ Cancer 2008;123:1864-70.

35. Kjaer SK, Munk C, Junge J, et al. Carcinogenic HPV prevalence and age-specific type distribution in 40,382 women with normal cervical cytology, ASCUS/LSIL, HSIL, or cervical cancer: what is the potential for prevention? Cancer Causes Control 2014;25:179-89.

36. Baldur-Felskov B, Dehlendorff C, Munk C, et al. Early impact of human papillomavirus vaccination on cervical neoplasia-nationwide follow-up of young Danish women. J Natl Cancer Inst 2014;106: djt460.

37. Mahmud SM, Kliewer EV, Lambert $P$, et al. Effectiveness of the quadrivalent human papillomavirus vaccine against cervical dysplasia in Manitoba, Canada. J Clin Oncol 2014;32:438-43. 